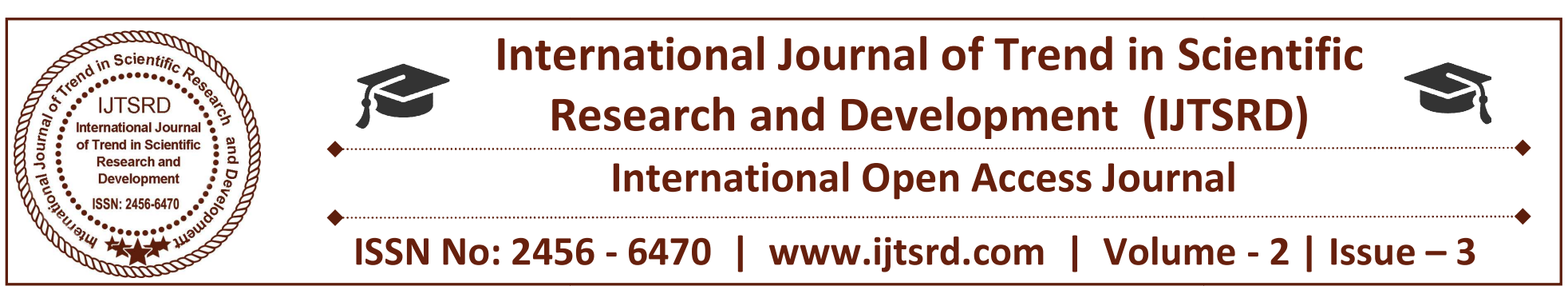

\title{
New Method for Making Digital Artifacts on Security Essentials of Web Verifiable and Reliable
}

A. Sai Haneesha

MCA Final Year, Lakireddy Bali Reddy College of

Engineering, Mylavaram, Andhra Pradesh, India

\author{
Ms. R. Vijaya \\ Associate Professor, Department of MCA, \\ Lakireddy Bali Reddy College of Engineering, \\ Mylavaram, Andhra Pradesh, India
}

\begin{abstract}
To make digital artifacts, for example, code, datasets, messages, and pictures obvious and changeless. Moreover, there is no regularly acknowledged strategy to authorize this changelessness of computerized curios. To take care of this issue, we propose trusty URIs containing base 64 encryption values. Base 64 encoding can be utilized while recognizing a substance which is utilized as a part of HTTP foundation.
\end{abstract}

Keywords: Digital Artifacts, Base64, Encoding, Immutable, HTTP

\section{INTRODUCTION}

Data mining is the extraction of data from huge databases which is al innovation which helps organizations centreon the most unmistakable data from their information stockrooms.

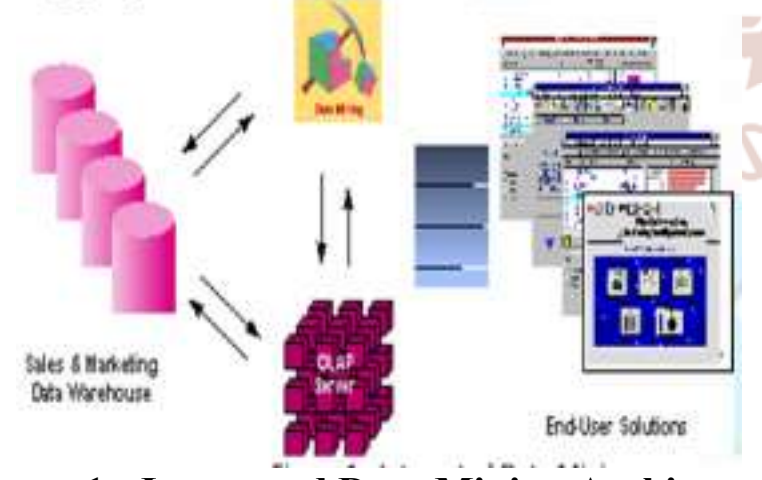

Figure 1 - Integrated Data Mining Architecture

Data mining gets its name from the association between looking for business information from a gigantic database - for example, finding associated data in gigabytes.
To apply the impelled techniques, data must be totally organized with a data appropriation centre and the business examination gadgets. A couple of information mining devices which work outside the circulation focus require extra intends to think and import. It requires operational utilization, Integration with the dispersion focus that reviews the utilization of results from data mining. The consequent coherent data stockroom can be associated in relationship, in domains, for the time being, campaign organization, and blackmail ID, for bleeding edge examination in a monster data appropriation focus. This conveyance focus can be completed in a grouping of social database systems should be redesigned for versatile and speedy data get to. Improvement of appropriation focus with new decisions and results, the affiliation can constantly complete the recommended systems and apply them to decisions. It is used for keeping up the database with generous course of action of aggregations which can be recouped successfully and enhances the benefit of information and is used to ensure that the customer conveys the data through URI which is tried and true or not. It is used to upgrade the confirmation of data which is effective while reusing the data.

\section{Back Ground}

In various locales and particularly in science, reproducibility is imperative. Evident, unchanging, and enduring mechanized relics are the basic segments for making the outcomes of robotized shapes reproducible; however the present Web offers no frequently recognized techniques to ensure these properties. Tries, for instance, the Semantic Web to 
appropriate complex learning in a machineinterpretable manner maddens this issue, as motorized calculations chipping away at tremendous measures of data can be required to be impressively more exposed than individuals to control or spoiled substance. Without fitting countermeasures, dangerous entertainers can destruct or trap such calculations by adding just absolutely controlled things to broad game plans of data

\section{PROPOSED SYSTEM}

This approach fuses cryptographic hash regards in the Web URI's, especially affirmation and decentralized outline. Our proposed approach comes down to references can be kept an eye if, despite everything that it contains a hash estimation of the referenced Digital trinket. This method does not have any critical bearing for each URIs, clearly, yet rather just to those which is to exhibit and changeless electronic collectible. We also propose trusty URI's for the web knick-knacks to be trustworthy and more secure.

Algorithm: Base64 encoding is used to perceive the information in a HTTP area. For instance, a database constancy framework may use Base64 encoding to encode a by and large broad fascinating id into a string for use as a HTTP parameter in HTTP structures or HTTP GET URLs. In like manner, various applications need to encode parallel data in a way that is favourable to be consolidated into URLs or covered web shape fields, and Base64 is a useful encoding to render them in a limited way.The calculation which is used as a piece of this module is changes over the ASCII motivating force to base64 String which offers security to the data that will be sent as a strong data. This keeps from unapproved interpreting of data.

\section{Significance of the Project}

The wander focuses in giving the substance of the data certain invariable and ceaseless with the objective that it can be used as a piece of various locales particularly in science since reproducibility is indispensable for making the outcomes of automated process. It makes the substance of the data tried and true which is sent as a URI to the customer and it guarantee whether it is trusted or not. It can be used generally in investigate centres where analysts disseminate research papers the papers are not to be changed yet rather to be copied and to watch that it is trusted data posted by the affirmed person.

\section{Module Description}

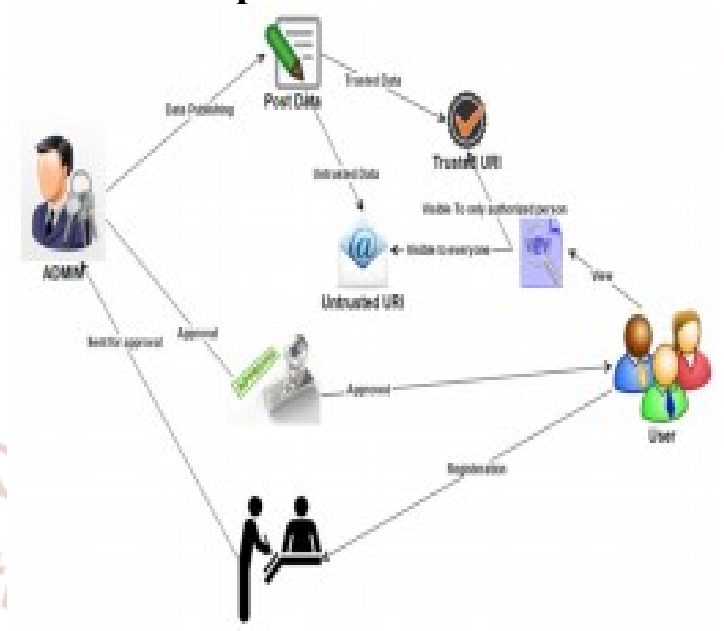

Figure 2-Architecture

Authentication and Authorization: Authentication is a technique in which the accreditations gave is checked to those on record in a database of endorsed customers' information in an area database or inside a confirmation server. Endorsement is the limit of demonstrating access to resources related to information security and PC security when all is said in done and to get the opportunity to control. In this endeavour affirmation is done to give more prominent security to the customers to have their own confirmations to sign in. The chairman supports the customers who are enrolled and offer rights to login to the method.

Cache of the Data: Cache which is for the most part used and incredibly unfaltering yet has not changed in years and is never again successfully made. The Cache is planned to help an architect in hanging on data for a foreordained time span. In this wander, it is used as the social affair of data to store which is used for various taking care of.

Secured Distribution (Encoding and Decoding): Encoding is the path toward making a progression of characters, for instance, letters, numbers, highlight, and certain pictures et cetera into a particular game plan for gainful transmission or limit. Deciphering is the contrary method - the difference in an encoded plan indeed into the main progression of characters. In Encoding, the data which are to be appropriated is being encoded and it is being changed into encoded regards and set away it in the database. In Decoding, 
the changed over data is being decoded back just if the honest to goodness customer enters else, it exhibits that you can't get to the record.

Distributing the data: Data disseminating is the route toward making the data open on the Internet, with the objective that they can be gotten to, separated and reused by anyone for investigate or distinctive purposes. The data is being circulated where the appropriate level has the agree to get to the archive which is controlled by the head.

\section{Data Flow}

\section{Level1: Login:}

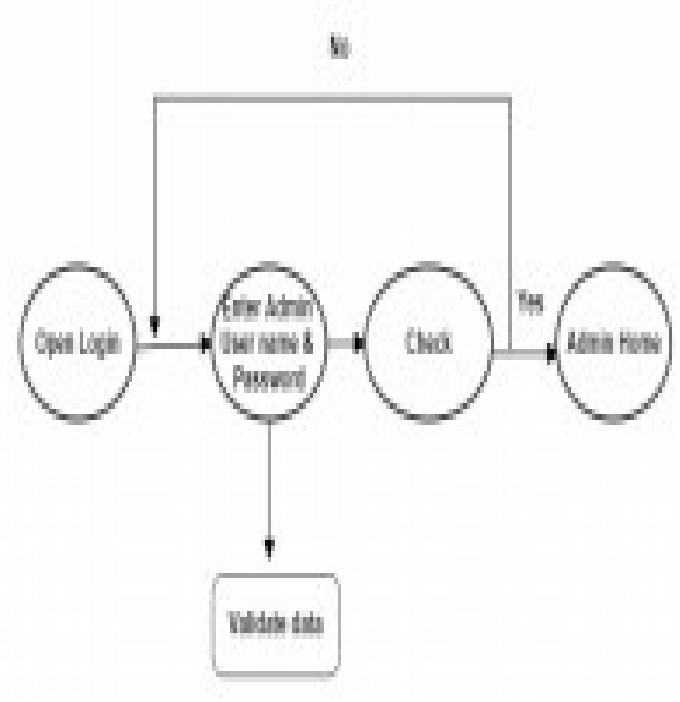

Figure 3-Login

\section{Level 2: Admin:}

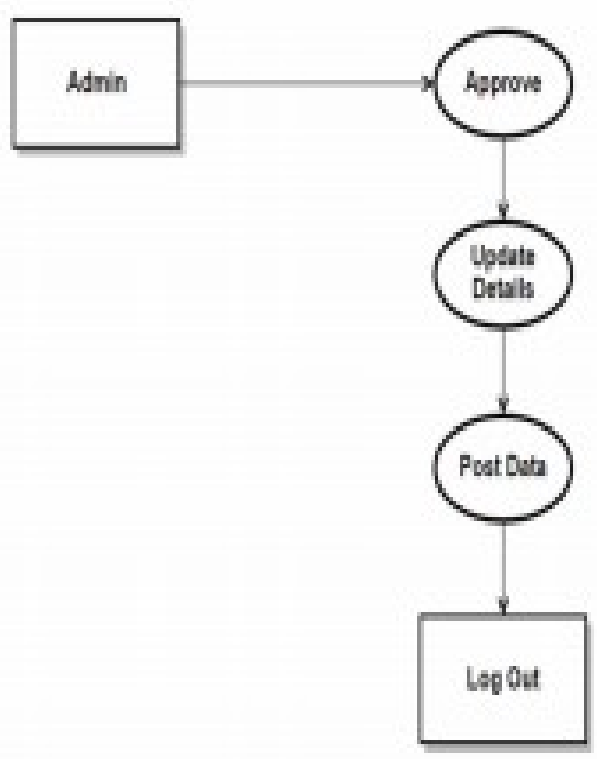

Figure 4-Admin

\section{Level 3:}

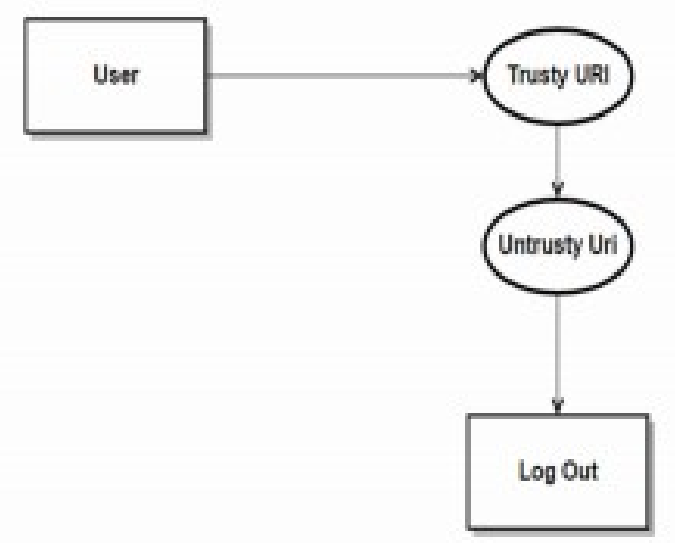

Figure 5-User

\section{CONCLUSION}

We have given a suggestion for express URI references to make modernized relics on the (Semantic) Web to be sure, invariable and enduring. In case got, it could impressively influence the structure and working of the Web, could enhance the profitability and exactness of gadgets using Web resources, which transforms into a basic specific section for the Semantic Web, especially for intelligent data, where provenance and sureness are vital. Further; we have started to develop a decentralized nanopublication server mastermind. Nanopublications are passed on and rehashed among such servers and recognized by trusty URIs, therefore ensuring that these antiquated rarities stay accessible paying little mind to whether solitary servers are finished. Additionally, we are tackling the possibility of nanopublication records that think about the definition and ID of little or enormous game plans of nanopublications.

\section{REFERENCES}

1. J. Carroll, "Signing RDF graphs," in Proc. 2nd. Semantic Web Conf., The Semantic Web, 2003, pp. 369-384.

2. E. H€ofig and I. Schieferdecker, "Hashing of RDF graphs and solution to the blank node problem," Proc. 10th Int. Workshop Uncertainty Reasoning Semantic Web, 2014, pp. 55.

3. M. Bellare, O. Goldreich, and S. Goldwasser, "Incremental cryptography: The hashing and signing," in Proc. 14th Annu. Int. Cryptol. Conf., Adv. Cryptol., 1994, pp. 216-233. 
4. M. Bellare, O. Goldreich, and S. Goldwasser, "Incremental cryptography: The hashing and signing," in Advances inCryptology CRYPTO'94. Springer, 1994, pp. 216-233.

5. C. Sayers and A. Karp, "Computing the digest of RDF graph,"Mobile and Media Systems Laboratory, HP Laboratories, PaloAlto, USA, Tech. Rep. HPL-2003-235(R.1), 2004.

6. R. Phan and D. Wagner, "Security considerations for incremental hash functions pair block chaining," Computers \&Security, vol. 25, no. 2, pp. 131-13, 2006.

7. C. Sayers and A. Karp, "Computing the digest of an RDF graph," Mobile and Media Systems Laboratory, HP Laboratories, Palo Alto, USA, Tech. Rep. HPL-2003-235(R.1), 2004.

8. R. Phan D. Wagner, "Security considerations for incremental hash functions based pair block chaining," Compute. Security, vol. 25, no. 2, pp. 131-136, 2006.

9. T. Kuhn and M. Dumontier, "Trusty URIs: Verifiable, immutable, permanent digital artifacts for linked data," in Proc. 11th Extended Semantic Web Conf., 2014, pp. 395-410.

10. P. Growth, A. Gibson, J. Velterop, "The anatomy of a nanopublication," Inf. Serv. Use, vol. 30, no. 1, pp. 51-56, 2010.

11. Farrell, Kutscher, C.Dannewitz, B.Ohlman, A.Keranen, Hallam-Baker, "Naming things with hashes," Internet Engineering Taskforce(IETF),StandardsTrackRFC6920,Apr.2 013.

12. R. Gentleman, "Reproducible research: A bioinformatics study," Statistical applications in genetics and molecular biology,vol. 4, no. 1, 2005.

13. F. Belleau, M. Nolin, N. Tourigny, P. Rigault, J. Morissette,"Bio2RDF: towards a mashup to build bioinformatics knowledgesystems," Journal Biomedical Informatics, vol. 41, no. 5, pp. 706716, 2008.

14. A. Callahan, J. Cruz-Toledo, and M. Dumontier, "Ontology-based querying Bio2RDF's linked data," Journal of BiomedicalSemantics, vol. 4, no. Suppl 1, p. S1, 2013.

15. T. Kuhn, C. Chichester, M. Dumontier, M. Krauthammer,"Publishing without publishers: a decentralized approach todissemination, retrieval, and archiving of data," arXiv preprintarXiv:1411.2749, 2014.
16. H. Van de Sompel, R. Sanderson, H. Shankar, and M. Klein,"Persistent identifiers scholarly assets and the web: The needfor an unambiguous mapping," International Journal of DigitalCuration, vol. 9, no. 1, pp. 331-342, 2014.

17. R. D. Peng, "Reproducible research computational science,"Science, vol. 334, no. 6060, p. 1226, 2011.

18. O. S. Collaboration et al., "An open, large-scale, collaborativeeffort estimate the reproducibilit psychological science,'Perspectives on Psychological Science, vol. 7, no. 6, pp. 657660, 2012.

\section{About Authors:}

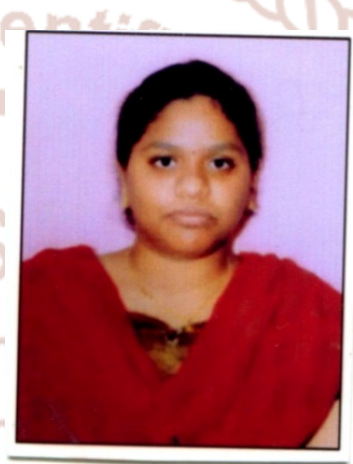

Ms.A.SAI HANEESHA is currently pursuing her MCA in LAKIREDDY BALIREDDY COLLEGE OF ENGINEERING, Mylavaram, A.P.

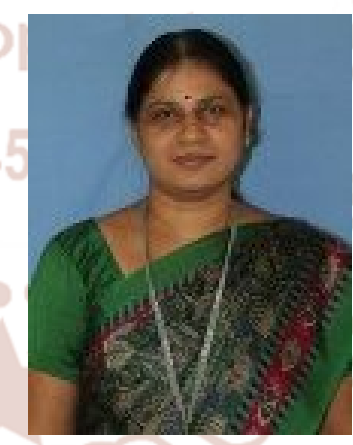

Ms.R.VIJAYA is currently working as an Associate Professor in MCA Department, LAKIREDDY BALIREDDY COLLEGE OF ENGINEERING, Mylavaram. 